\title{
A NEW OPERATION FOR THE TREATMENT OF HIRSCHSPRUNG'S DISEASE*
}

BY

\author{
BERNARD DUHAMEL \\ From Paris
}

The excision of the aganglionic segment of the recto-sigmoid colon proposed by Swenson is the logical outcome of the new conception of the aetiology of Hirschsprung's disease and I do not think it necessary to discuss it here any further.

The operation which I shall describe constitutes but a technical variance of Swenson's operation and is not a new method; it has also some other applications apart from the treatment of congenital megacolon. The object of this operation is to exclude and not to resect the malformed rectum and all dissection of the pelvis is avoided. The functionally healthy proximal colon is brought down to the margin of the anus in a plane of anatomical cleavage, and thus the nervous system of the bladder and neuro-muscular apparatus of the sphincters are preserved in toto. A wide anastomosis between the colon and the excluded rectum is performed and, by preserving the rectum, an important area of reflex activity is preserved.

Hirschsprung's disease is a serious condition but it is not a malignant disease in the usual sense of the term; therefore an operation designed to remedy the condition should not be of excessive severity, and there should be no risk that one functional anomaly (urinary incontinence or impotence) will be substituted for another. Swenson's operation is a delicate and difficult intervention which only gives excellent results if it is carried out by a very competent surgeon operating on children of a certain age.

Since the characteristic deficiency of ganglia in Hirschsprung's disease nearly always affects the whole of the rectum down to the level of the internal sphincter, an extensive resection of the rectum, which is associated with considerable shock, especially in the newly born, must be performed in order to obtain a satisfactory surgical result.

Dissection of the rectum can be carried out close to the muscular layer since one is not dealing with a malignant condition; it is nevertheless possible to traumatize the pelvic nervous plexus supplying

\footnotetext{
* A paper read at a meeting of the British Association of Paediatric Surgeons held in Liverpool in June, 1959.
}

the bladder or the genitals. Complete ablation of the mucous membranes of the rectum or of its nerve supply will interfere with rectal sensation which is indispensable to the perfect function of the sphincters.

Whatever the extent of the resection of the rectum, it is always incomplete inferiorly as it is essential to preserve the rectal sphincter. Thus an unsatisfactory result is common and relapses are possible.

The end-to-end anastomosis is liable to separate either partially or completely if the vitality of the pulled-down colon is endangered or if the operative technique is incorrect. In a certain number of cases serious complications, such as peritonitis, pelvic abscess or fistula, may result.

Lastly the anastomosis can (and this is a most frequent complication) become stenosed and may at times become completely occluded.

It is because of these complications, which cannot be avoided in spite of the technical modifications described by some authors, that some surgeons have rejected Swenson's operation and returned to abdominal resection followed by dilatation of the sphincter. This is probably a retrograde step since one knows that in most cases the lower part of the rectum and the internal sphincter are themselves malformed. The same objections could be raised against those who have proposed a recto-sigmoidmyotomy inspired by the operation of Heller since they do not prolong their extramucous incision beyond the level of the pouch of Douglas, and have to complete the operation by carrying out an anal dilatation.

In a recent book (Duhamel, 1957) I have described and illustrated in detail my surgical technique and I shall therefore mention here only the essential steps.

\section{Operative Procedure}

Abdominal Approach. (1) The rectum is divided, closed and buried at the level of the pouch of Douglas, as in Hartman's operation.

(2) After resection of the narrowed rectosigmoidal zone and that of the adjoining megacolic 
segment, which is usually toneless and full of faecoliths, the proximal colon is mobilized (care being taken to preserve its blood supply) and must be long enough to allow its being brought down to the anus; its lower end is temporarily closed using a few large sutures.

(3) The cellular retro-rectal space is now opened by cutting through the meso-rectum and dissection is carried downwards in the midline using the finger, the hand or a swab on a holder until the level of the pelvic diaphragm is reached.

Perineal Approach. (4) The anus is dilated and an incision is made along its posterior circumference at the line of the ano-cutaneous junction. The wall of the anal canal is then dissected off the external sphincter and after a few muscle fibres have been split longitudinally at the upper border of the external sphincter one meets the retro-rectal dissection of the abdominal approach.

(5) With the aid of a curved clamp introduced through this opening, the resected lower end of the colon is grasped and pulled down to the level of the skin incision. The lower end of the colon is reopened and its posterior margin is sutured to the posterior cutaneous lip of the retro-anal incision.

(6) Two Kocher's forceps are inserted, one blade into the lower colon, the other into the rectum; their points meet in a $\mathrm{V}$ high up in the bowel and their bases are held apart so that they come to lie at the lateral angle of the colon and anus. The forceps are tightened and then tied together. The colon cannot now retract and the forceps produce haemostasis. After a few days a longitudinal enterostomy opening between the colon and the rectum is produced. The newly fashioned rectum has an anterior part which is aganglionic, reflex control of defaecation thus being assured, and a posterior part composed of colon which will ensure mobility.

I have operated on 11 cases of Hirschsprung's disease using this technique; this is obviously too small a series for statistical purposes but I know that some French, Spanish, Finnish and Swiss surgeons have adopted this operation with results similar to mine. In every case we have been struck by a complete absence of operative shock and by the fact that spontaneous stools are passed within two to four days. Cutting out of the enterostomy forceps takes about a week followed by scarring without com- plications. The functional result has always been perfect and radiological studies have shown complete regression of colonic dilatation and a normal diameter after an interval of about two to four months.

All these cases show an improvement as good as those operated on by Swenson's method, but I realize that this alone will not convince experienced surgeons who are anyway getting regularly satisfactory results. There are however two particular aspects of the operation which I think constitute a distinct improvement on the old technique. Firstly, this operation is the only one which allows re-operation on cases of 'failed' Swenson's operation, both in failures due to insufficient resection of the lower segment and in those with stenosis of the anastomosis. This advantage has already been recognized by numerous surgeons. Secondly, the most important advantage of the operation is that it allows a considerable lowering of the age limit at operation. Most authors agree that Swenson's operation is dangerous in the newly born because of the difficulties encountered in dissecting the rectum at that age. Swenson himself advises against recto-sigmoidectomy until the child is at least 12 to 18 months old, and suggests that, if necessary, a temporary colostomy only should be carried out.

When recto-sigmoidectomy is performed during the first months of life, the results are often unsatisfactory because in order to save a sufficient amount of gut for proper function of the anal reflexes one is forced to preserve too much of the internal sphincter and it is often necessary to perform a complementary sphincterotomy. The technique that I have described overcomes these disadvantages. I have operated on eight children of less than 6 months of age, five of whom were less than 3 months old, and I have noticed that the younger the child the better have been the operative results and the quicker the return to normal function. Realizing the particular seriousness of congenital megacolon in the neonatal period, and the dangers of a temporary colostomy at this age, we believe that the introduction of a curative operation for Hirschsprung's disease in infancy will allow us to save more children suffering from the so-called 'malignant' form of the disease in the neo-natal period.

Duhamel, B. (1957). Technique Chirurgicale Infantile. Masson, 